\title{
Long-time self-diffusion in quasi-two-dimensional colloidal fluids of paramagnetic particles
}

\author{
Nima H. Siboni $\odot,{ }^{1}$ Alice L. Thorneywork, ${ }^{2,3}$ Alicia Damm $\odot,{ }^{2}$ Roel P. A. Dullens, ${ }^{2}$ and Jürgen Horbach ${ }^{4}$ \\ ${ }^{1}$ Institut für Theoretische Physik, Technische Universität Berlin, Hardenbergstraße 36, 10623 Berlin, Germany \\ ${ }^{2}$ Department of Chemistry, Physical and Theoretical Chemistry Laboratory, University of Oxford, \\ South Park Road, Oxford OX1 3QZ, United Kingdom \\ ${ }^{3}$ Cavendish Laboratory, University of Cambridge, Cambridge CB3 OHE, United Kingdom \\ ${ }^{4}$ Institut für Theoretische Physik II, Heinrich-Heine-Universität Düsseldorf, Universitätsstraße 1, 40225 Düsseldorf, Germany
}

(Received 16 October 2019; accepted 3 April 2020; published 24 April 2020)

\begin{abstract}
The effect of hydrodynamic interactions (HI) on the long-time self-diffusion in quasi-two-dimensional fluids of paramagnetic colloidal particles is investigated using a combination of experiments and Brownian dynamics (BD) simulations. In the BD simulations, the direct interactions (DI) between the particles consist of a shortranged repulsive part and a long-ranged part that is proportional to $1 / r^{3}$, with $r$ the interparticle distance. By studying the equation of state, the simulations allow for the identification of the regime where the properties of the fluid are fully controlled by the long-ranged interactions, and the thermodynamic state solely depends on the dimensionless interaction strength $\Gamma$. In this regime, the radial distribution functions from the simulations are in quantitative agreement with those from the experiments for different fluid area fractions. This agreement confirms that the DI in the experiments and simulations are identical, which thus allows us to isolate the role of $\mathrm{HI}$, as these are not taken into account in the BD simulations. Experiment and simulation fall onto a master curve with respect to the $\Gamma$ dependence of $D_{\mathrm{L}}^{\star}=D_{\mathrm{L}} /\left(D_{0} \Gamma^{1 / 2}\right)$, with $D_{0}$ the self-diffusion coefficient at infinite dilution and $D_{\mathrm{L}}$ the long-time self-diffusion coefficient. Our results thus show that, although $\mathrm{HI}$ affect the short-time selfdiffusion, for a quasi-two-dimensional system with $1 / r^{3}$ long-ranged DI, the reduced quantity $D_{\mathrm{L}}^{\star}$ is effectively not affected by HI. Interestingly, this is in agreement with prior work on quasi-two-dimensional colloidal hard spheres.
\end{abstract}

DOI: 10.1103/PhysRevE.101.042609

\section{INTRODUCTION}

Colloidal experiments have been very important for the understanding of the dynamics and phase behavior of twodimensional (2D) systems. Examples are experiments on the nature of the two-stage melting scenario [1-5], the structure and diffusion in 2D fluids [6-19], the diffusion through 2D random energy landscapes and porous media [20-24], or the glass transition in 2D [25-32]. These experimental studies have been performed on well-characterized model systems in a quasi-2D geometry, which is realized by either sedimenting a monolayer of colloidal particles on a glass plate [13-17], confining the particles between closely spaced glass plates $[8,9,33,34]$, or suspending them on the waterair interface of a water drop [1-3]. As a consequence, the colloidal particles move in a 2D plane and, in the case of hard spheres, one effectively obtains a hard-disk system $[5,13]$.

Experimental colloidal fluids in quasi-2D geometry have been extensively studied and are well characterized with respect to structural and thermodynamic properties. Experimental realizations of quasi-2D hard-sphere fluids have shown excellent agreement with theory and simulation of corresponding 2D hard-disk systems with regard to static structure factors, the equation of state, and the dependence of various static susceptibilities (e.g., the isothermal compressibility) on density [5,13,17]. Similar agreement between theory/simulation and experiment has been also shown for other quasi-2D fluid systems, e.g., in the framework of experiments of paramagnetic colloids on a water-air interface $[7,10,11]$. In paramagnetic fluids, the direct interaction between particles is controlled-in addition to their hard core-by a long-range potential, that decreases as $1 / r^{3}$ with $r$ the distance between a pair of particles. In the case of binary 2D mixtures of paramagnetic particles of different size, a microphase structuring has been found which is associated with a "clustering" of the smaller particles $[10,11]$. The latter examples show that static properties of both hard-disk and paramagnetic 2D fluids are well understood; in particular, quantitative agreement with colloid experiments in quasi-2D geometry is obtained.

Less clear is the situation with respect to dynamic quantities such as the short- and long-time self-diffusion coefficients in colloidal quasi-2D fluids. Here, it is not well understood how the diffusive behavior is affected by hydrodynamic interactions (HI) due to the momentum transport through the solvent in which the colloids are suspended [35]. While the direct particle interactions are restricted to a $2 \mathrm{D}$ plane, the solvent is not restricted to this plane and thus momentum can be transported away from this plane. There have been several theoretical and experimental studies [6,14,36-38] on the influence of HI on the long-time self-diffusion coefficient, $D_{\mathrm{L}}$, in quasi-2D geometry. Here, theories for hard-sphere systems in quasi-2D geometry have suggested that HI make the long-time self-diffusion slower [38]; however, the latter picture has recently been challenged [14]. More specifically, using a combination of simulation and experiment, it has been shown for hard-sphere fluids in quasi-2D geometry that over 
a wide range of fluid densities the ratio $D_{\mathrm{L}} / D_{0}$ (with $D_{0}$ the self-diffusion coefficient at infinite dilution) is effectively not affected by HI. In contrast to this, for fluids of paramagnetic particles, it has been reported that HI accelerate the longtime self-diffusion in experiments [6]; an observation that has also been supported by theoretical studies based on the Rotne-Prager approximation of the hydrodynamic diffusion tensor [36,37].

In this paper, we readdress the question as to whether the interplay of HI and direct interactions is fundamentally different in quasi-2D colloidal systems if the direct interactions between the colloidal particles are long-ranged. To this end, we use a methodology similar to that in our recent work on hard-core systems [14], and compare quasi-2D colloidal experiments on paramagnetic particles to Brownian dynamics (BD) simulations of corresponding 2D systems where HI are not taken into account. We first identify, from the BD simulations, the regime in which the long-ranged $1 / r^{3}$ component of the interaction dominates by a careful study of the equation of states (EoS), and show that the experimental states considered - as characterized by the coupling parameter $\Gamma$-correspond to this regime. Next, we compare the radial distribution functions obtained from experiment with those from the BD simulation. We find quantitative agreement between experiment and simulation with respect to the structure for all states considered, indicating that the direct interactions are the same in simulation and experiment. The dynamics of the system are then investigated via the mean-squared displacement (MSD) from which we determine the short- and long-time self-diffusion coefficients. We find that while the short-time self-diffusion coefficient slightly differs from the simulation values, indicating the role of $\mathrm{HI}$ at short times, the reduced long-time self-diffusion coefficient $D_{\mathrm{L}}^{\star}=D_{\mathrm{L}} / D_{0} \Gamma^{1 / 2}$ from experiment is in excellent agreement with that from the simulations. Furthermore, $D_{\mathrm{L}}^{\star}$ is solely a function of the reduced density $\Gamma^{2 / 3}$ (see below), and all the data for the longtime self-diffusion coefficient from simulation and experiment fall onto a master curve when $D_{\mathrm{L}}^{\star}$ is plotted as a function of $\Gamma^{2 / 3}$. We show that this behavior is expected for the BD of a fluid where the particles interact via a $1 / r^{3}$ potential, of course without being affected by HI.

\section{COLLOID EXPERIMENTS}

The quasi-2D colloidal system consists of carboxylic acid functionalized paramagnetic polystyrene spheres (Dynabeads, Invitrogen) with a hard sphere diameter of $\sigma_{\mathrm{hs}}=3.16 \mu \mathrm{m}-$ obtained from a mapping to density functional theory [13]dispersed in a 20/80 v/v\% ethanol-water mixture. The particles sediment onto the base of a glass sample cell with a height of $200 \mu \mathrm{m}$ to form a monolayer, and as their gravitational length is $0.07 \mu \mathrm{m}$, the out-of-plane fluctuations are negligible relative to the particle diameter in the absence of an external magnetic field [13].

Upon the application of an external magnetic field perpendicular to the plane of the particles, a dipole moment is induced, $\mathbf{m}=\chi \mathbf{B}$, where $\chi$ is the magnetic susceptibility of the particles and $\mathbf{B}$ is the magnetic field which is perpendicular to the plane of particles. The well-defined hard core area fraction $\phi$ is given $\phi=\pi \sigma_{\mathrm{hs}}^{2} N /(4 A)$, where $\sigma_{\mathrm{hs}}$ is the hard sphere

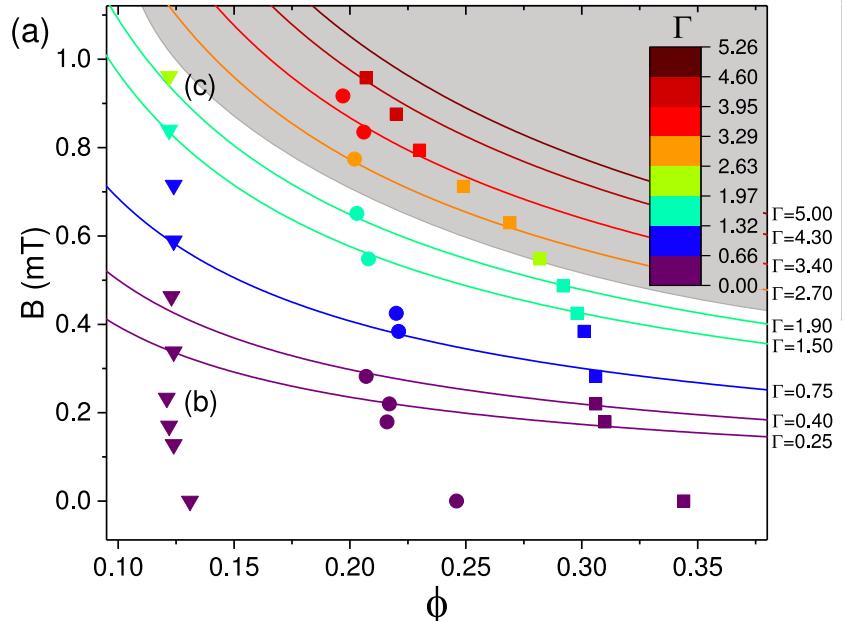

(b)

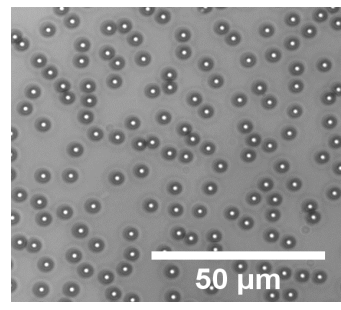

(c)

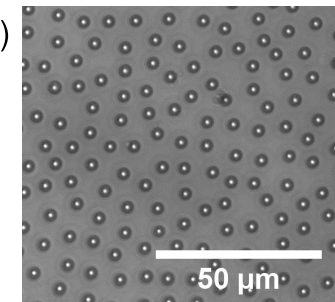

FIG. 1. (a) State diagram for the experiments in the $(B, \phi)$ plane. The solid lines are isolines, corresponding to constant values of $\Gamma$ (the colors of the lines indicate the value of $\Gamma$ according to the color map). The shaded area represents states where out-of-plane stacking of some colloidal particles is observed, leading to a reduction of the (in-plane) area fraction. (b) and (c) Snapshots of the colloidal system at (b) $\phi \simeq 0.12$ and $B \simeq 0.23 \mathrm{mT}$, corresponding to $\Gamma \simeq 0.11$, and (c) $\phi \simeq 0.12$ and $B \simeq 0.96 \mathrm{mT}$, corresponding to $\Gamma \simeq 1.96$.

diameter, $N$ is the number of particles, and $A$ is the area of the system imaged. Considering the nature of the interactions, a single dimensionless interaction energy-also termed the coupling parameter-for the relevant energies can be defined as the ratio of the magnetic dipole-dipole interaction energy and the thermal energy:

$$
\Gamma=\mu_{0} m^{2} /\left(k_{\mathrm{B}} T 4 \pi \tilde{r}^{3}\right)
$$

with $\mu_{0}$ the vacuum permeability and $\tilde{r}$ the average interparticle distance, $\tilde{r}=\sqrt{A / N}$. The coupling parameter $\Gamma$ is a reduced inverse thermal energy. Its role as a control parameter for the thermodynamic state as well as the dynamics of the system will be discussed in more detail below.

The experimentally explored values of $\phi$ and $B$, as well as the corresponding $\Gamma$, are shown in Fig. 1(a). We note that, upon increasing the magnetic field, particles can leave the monolayer and form a second layer by stacking on top of each other. This phenomena becomes increasingly significant for high packing fractions and/or high magnetic fields and, as such, only relatively low values of $\phi$ are considered here. In spite of this, for a few samples we do observe a small number of out-of-plane particles, and the range of parameters associated with this is indicated by the shaded area in Fig. 1(a). The colloidal system is imaged using video microscopy and the particle positions are acquired using standard particle tracking 
software [39]. Typical snapshots of the system at two different values of $\Gamma$ are shown in Figs. 1(b) and 1(c).

\section{SIMULATIONS}

BD simulations are performed for 2D systems that consist of $N=400$ particles, using periodic boundary conditions in the two Cartesian directions. The particles interact via (i) a magnetic dipole-dipole interaction potential,

$$
u_{\text {dip }}(r)=A_{\text {dip }} / r^{3}
$$

with $A_{\text {dip }}=\mu_{0} m^{2} /(4 \pi)$ controlling the strength of the potential and $r$ the distance between a pair of particles, and (ii) a short-ranged repulsive potential, given by

$$
u_{\mathrm{rep}}(r)=4 \epsilon\left[\left(\frac{\sigma}{r}\right)^{48}-\left(\frac{\sigma}{r}\right)^{24}+\frac{1}{4}\right],
$$

for $r \leqslant 2^{1 / 24} \sigma$ and zero elsewhere. This potential provides a good model of the hard core of the paramagnetic particles (see, e.g., Ref. [40]).

The potential $u_{\text {dip }}$ is a slowly decaying function which requires the use of the 2D Ewald summation technique [41] to accurately compute the potential energy, the forces, and the pressure. The units used in the simulation for length and energy are given by $\sigma$ and $\epsilon$, respectively. Time is given in units of $\tau_{\text {sim }}=\sqrt{m \sigma^{2} / \epsilon}$, with $m=1.0$ the mass of a particle. Thus, the amplitude $A_{\text {dip }}$ is given in units of $\epsilon \sigma^{3}$ and the dimensionless parameter $\Gamma$ can be expressed as

$$
\Gamma=\beta A_{\text {dip }} / \tilde{r}^{3}=\beta A_{\text {dip }} \rho^{3 / 2}
$$

with $\beta=1 /\left(k_{B} T\right)$. Note that this is a generalized form of Eq. (1) for $\Gamma$, which corresponds to a reduced inverse thermal energy.

In the Ewald summation, we have chosen $\alpha_{\mathrm{Ew}}=0.5 \sigma^{-1}$ for the Ewald constant. The real space cutoff in the Ewald summation is always set to half the size of the system (note that in the considered systems the linear dimension of the "simulation box" varies between $15 \sigma$ and $25 \sigma$ ). The cutoff in the Fourier-space sum is set such that $G_{\mathrm{Ew}}>10 \sigma^{-1}$ holds for the absolute value of the largest considered wave vector.

The dynamics of the system are described by over-damped Brownian motion. The equations of motion are discretized using a forward Euler scheme given by $[42,43]$

$$
\mathbf{r}_{i}(t+\delta t)=\mathbf{r}_{i}(t)+\frac{D_{0}}{k_{\mathrm{B}} T} \mathbf{F}_{i}(t) \delta t+\sqrt{2 D_{0} \delta t} \boldsymbol{\xi}_{i},
$$

with $\delta t$ the integration time step, $\mathbf{r}_{i}(t)$ the position of particle $i$ at time $t, \mathbf{F}_{i}(t)$ the force on particle $i$ due to the direct particle interactions, $D_{0}$ the self-diffusion coefficient at infinite dilution, and $\xi_{i}$ a vector with independent Gaussian random numbers with zero mean and unit variance (note that one can use uniform random numbers instead of Gaussian distributed ones, as shown by Dünweg and Paul [44]). The code to solve Eq. (5) has been implemented in the LAMMPS package [45]. In our simulation, we have set $D_{0}=1.0 \sigma^{2} / \tau_{\text {sim }}, k_{\mathrm{B}} T=0.5 \epsilon$ and integrated the equations of motion with a time step of $\delta t=5 \times 10^{-5} \tau_{\text {sim }}$.

To demonstrate that Ewald sums are in fact necessary for a reliable evaluation of the long-ranged dipole-dipole interactions, we show in Fig. 2 the "excess" pressure $P_{\text {ex }}$

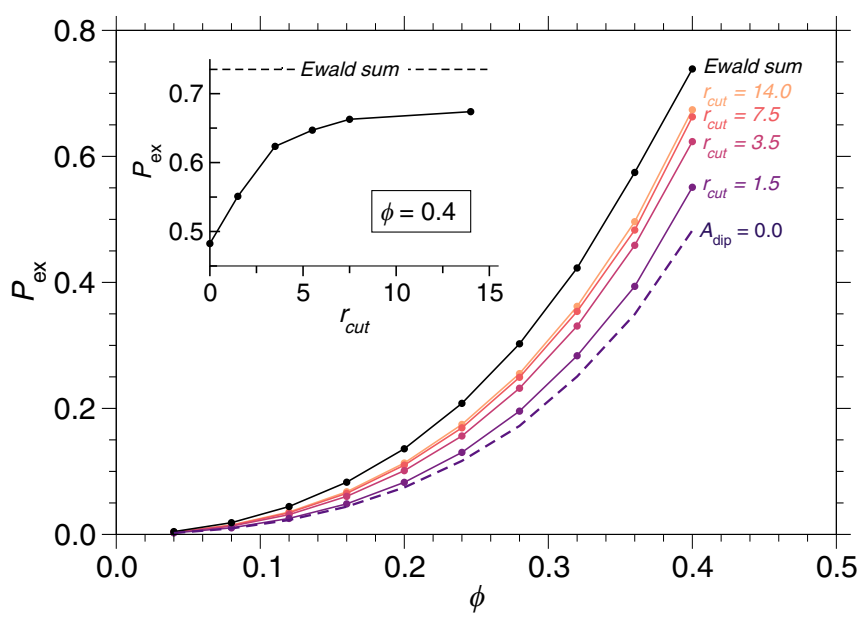

FIG. 2. Comparison of the excess pressure $P_{\mathrm{ex}}$ as function of $\phi$, as obtained from the simulation with Ewald summation, to that obtained by employing different cutoff radii $r_{\text {cut }}$ in $u_{\text {dip }}(r)$. The amplitude of the long-ranged $1 / r^{3}$ potential is set to $A_{\text {dip }}=0.175561$. As a reference, the dashed line shows the case $A_{\text {dip }}=0$ where the $1 / r^{3}$ interaction is switched off. The inset displays the dependence of $P_{\text {ex }}$ on $r_{\text {cut }}$ for $\phi=0.4$, which indicates a slow convergence of $P_{\text {ex }}$ with increasing $r_{\text {cut }}$. Here, the value of $P$, as obtained via Ewald summation, is marked as a horizontal dashed line.

[see Eq. (8) below as a function of the area fraction $\phi$, i.e., the EoS, as obtained from simulations for $A_{\text {dip }}=0.175561$ with Ewald summation in comparison to simulations where the potential $u_{\text {dip }}(r)$ is cut off at a radius $r_{\text {cut }}$. Also included in this plot is a curve marked with $A_{\text {dip }}=0$, corresponding to the case where the potential $u_{\text {dip }}(r)$ is switched off. The plot indicates the slow convergence of $P_{\mathrm{ex}}$ with increasing $r_{\text {cut }}$, especially at large packing fractions. The inset of Fig. 2 displays $P_{\text {ex }}$ as function of $r_{\text {cut }}$ at $\phi=0.4$. It shows that even for the highest considered cutoff $r_{\text {cut }}=14$ the value of the pressure is about $10 \%$ below the "exact" value, as obtained from the Ewald summation (dashed horizontal line). Thus, for a reliable determination of the EoS, presented below, the use of Ewald summation is indispensable.

\section{RESULTS AND DISCUSSION}

\section{A. Equation of state}

For a system with a long-ranged potential according to Eq. (2), i.e., $\sim 1 / r^{3}$, which corresponds to point dipoles, the thermodynamic state can be uniquely defined by $\Gamma$ [Eq. (4)]. As a result, reduced thermodynamic quantities as a function $\Gamma$ will fall onto a master curve, irrespective of whether $\Gamma$ is changed by varying $B$ or $\rho$. However, in the system considered here the particles also interact via the short-ranged repulsive potential, given by Eq. (3), which models the hard-core interaction between the particles in the experimental system. This hard-core potential is important for systems where the distance between neighboring particles is of the order of $\sigma$. Importantly, if this is the case, the thermodynamic state is no longer uniquely defined by $\Gamma$ and one expects deviations from the master curve for a thermodynamic quantity. By determining where these deviations do not occur, it is possible 
to establish the regime where the behavior of the system is dominated by the long-ranged dipolar $1 / r^{3}$ repulsion.

We first review the scaling properties of systems that consist of particles interacting with each other via a pair-wise additive inverse power potential. Consider a classical system of $N$ particles in a $D$-dimensional volume $V$, where the particles interact via $u(r)=\epsilon\left(\frac{\sigma}{r}\right)^{n}$, with $n \in \mathbb{N}, \epsilon$ an energy, and $\sigma$ a length. For such systems, thermodynamic properties do not separately depend on temperature $T\left[\right.$ or $\left.\beta=1 /\left(k_{\mathrm{B}} T\right)\right]$ and number density $\rho=N / V$, but on a combination of these two variables. This can be easily seen if one considers the Boltzmann factor $\exp \left(-\beta \sum_{i=1}^{N} \sum_{j>i} u\left(r_{i j}\right)\right)$, where $\sum_{i=1}^{N} \sum_{j>i}$ denotes the double sum over all $\frac{1}{2} N(N-1)$ particle pairs and $r_{i j}=\left|\mathbf{r}_{i}-\mathbf{r}_{j}\right|$ is the distance between particle $i$ and particle $j$. In terms of scaled coordinates, $\mathbf{r}_{i}^{\star}=\rho^{1 / D} \mathbf{r}_{i}$, the Boltzmann factor can be written as $\exp \left(-\beta^{\star} \sum_{i=1}^{N} \sum_{j>i}\left(\frac{1}{r_{i j}^{\star}}\right)^{n}\right) \equiv$ $\exp \left(-\beta^{\star} U^{\star}\right)$, introducing the reduced inverse thermal energy $\beta^{\star}=\beta \epsilon \sigma^{n} \rho^{n / D}$. Note that a reduced density can be defined as $\rho^{\star}=(\beta \epsilon)^{D / n} \sigma^{D} \rho$. As a consequence, canonical averages $\langle\cdots\rangle$ of a quantity $A\left(\mathbf{r} \equiv\left(\mathbf{r}_{1}, \ldots, \mathbf{r}_{2}\right)\right)$ can be written as an average of a reduced quantity $A^{\star}\left(\mathbf{r}^{\star}\right)$ [46-48],

$$
\langle A(\mathbf{r})\rangle=\rho^{-\alpha / D} \tau^{\theta} m^{\gamma}\left\langle A^{\star}\left(\mathbf{r}^{\star}\right)\right\rangle_{\star}
$$

with

$$
\left\langle A^{\star}\left(\mathbf{r}^{\star}\right)\right\rangle_{\star}=\frac{\int d \mathbf{r}^{\star} A^{\star}\left(\mathbf{r}^{\star}\right) \exp \left(-\beta^{\star} U^{\star}\right)}{\int d \mathbf{r}^{\star} \exp \left(-\beta^{\star} U^{\star}\right)} .
$$

The average $\left\langle A^{\star}\left(\mathbf{r}^{\star}\right)\right\rangle_{\star}$ depends only on the reduced variable $\beta^{*}$ or, alternatively, on the reduced density $\rho^{\star}$. In Eq. (6), $m$ is the mass of a particle and the timescale $\tau$ is defined by [48] $\tau=\left(m \rho^{-(n+2) / D} / \epsilon \sigma^{n}\right)^{1 / 2}$. The exponents $\alpha, \theta$, and $\gamma$ in Eq. (6) are chosen according to the units of the quantity $A(\mathbf{r})$.

For our system, we now consider the reduced pressure as a function of the reduced density $\rho^{*} \equiv \Gamma^{2 / 3}=\left(\beta A_{\text {dip }}\right)^{2 / 3} \rho$. To this end, we start with the excess pressure, which is defined by

$$
P_{\mathrm{ex}}=P-\frac{\rho}{\beta}=\frac{n}{D V}\left\langle\sum_{i=1}^{N} \sum_{j>i} \epsilon\left(\frac{\sigma}{r_{i j}}\right)^{n}\right\rangle
$$

with $P$ the total pressure. A reduced pressure, $P^{\star}\left(\rho^{\star}\right)$, can be defined using Eq. (6) via $P=P^{\star} m \rho^{1-2 / D} / \tau^{2}=$ $P^{\star} \in \sigma^{n} \rho^{1+n / D}$, and thus $\beta P / \rho=P^{\star} \rho^{\star n / D}$ (note that we can consider the total pressure because by the scaling with $\beta / \rho$ the ideal gas contribution is just equal to 1). Applying this to a $2 \mathrm{D}$ system with long-ranged dipole-dipole interactions $\propto r^{-3}$, Eq. (2), we can first identify $\epsilon=A_{\text {dip }} / \sigma^{3}$ and thus $\rho^{\star}=\rho\left(\beta A_{\mathrm{dip}}\right)^{2 / 3}=\Gamma^{2 / 3}$. Therefore, the reduced pressure is given by $P^{\star}\left(\Gamma^{2 / 3}\right)=\frac{\beta P}{\rho} \Gamma^{-1}$ which is solely a function of the reduced density $\Gamma^{2 / 3}$.

To explore the relative importance of the long-ranged dipolar and hard-core parts of the potential and thereby identify the experimental regime where the interactions are dominated by the $1 / r^{3}$ repulsion, we determine the reduced pressure $P^{\star}$ as a function of $\rho^{*} \equiv \Gamma^{2 / 3}$, for simulated systems at different fixed values of $A_{\text {dip }}$ - corresponding to the magnetic field $B$ in the experiments - and varying $\rho$. In Fig. 3 , we show the reduced

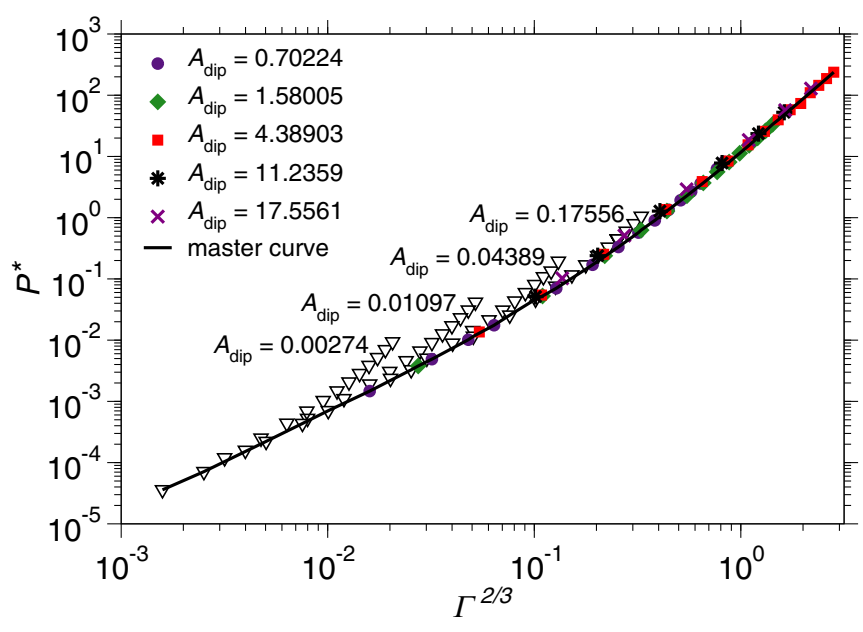

FIG. 3. The reduced pressure $P^{\star}$ as a function of $\rho^{*} \equiv \Gamma^{2 / 3}$ for different fixed values of $A_{\text {dip }}$ and varying $\rho$. States corresponding to low values of $A_{\text {dip }}$, i.e., for $A_{\text {dip }} \leqslant 0.17556$, are represented by open triangles. Also shown is a master curve (solid line), which corresponds to the behavior of point dipoles. For states on this master curve, the effect of the hard-core part of the potential is negligible and the particle interactions are dominated by the dipole-dipole part of the potential, as given by Eq. (2).

pressure $P^{\star}$ as a function of $\Gamma^{2 / 3}$ for different values of $A_{\text {dip }}$. For a small value of $A_{\text {dip }}$ such as $A_{\text {dip }}=0.00274$, the reduced pressure follows a master curve (shown as a solid line) for small values of $\Gamma^{2 / 3}$, corresponding to sufficiently low densities such that the interparticle distance is significantly larger than $\sigma$. For higher values of $\Gamma^{2 / 3}$ and thus higher densities, the particles feel the repulsive short-ranged potential (3) and, as a consequence, the reduced pressure deviates from the master curve. With increasing $A_{\text {dip }}$, one increases the strength of the long-ranged dipolar interactions, and therefore the point above which one expects deviations from the master curve shifts to larger values of $\Gamma^{2 / 3}$. As Fig. 3 indicates, for the considered states for $A_{\text {dip }}>0.17556$ (filled symbols), all the points collapse onto the master curve. Note that the experiments were done for similar states in the latter regime (corresponding to states with $B>0.1 \mathrm{mT}$ in Fig. 1) and so, with respect to the considered states in the experiments, only dipole-dipole interactions are relevant.

\section{B. Radial distribution functions: Comparison between simulation and experiment}

The analysis in the previous section shows that the states considered in the experiment (cf. Fig. 1) are in a regime where the interactions between the particles are controlled by the long-ranged dipole-dipole interactions $\propto r^{-3}$. As such, the interaction between a pair of colloidal particles in experiment should be given by the potential, Eq. (2), and we expect a close agreement between the structural correlations found in the experiments and those in the simulations. To check this, we compute the radial distribution function $g(r)$ that describes how structural correlations vary, relative to an ideal gas, as a function of the distance $r$ from a reference particle. It is 
defined by [49]

$$
g(r)=\frac{A}{2 \pi r} \frac{1}{N^{2}}\left\langle\sum_{i=1}^{N} \sum_{j=1, \neq i}^{N} \delta\left(r-r_{i j}\right)\right\rangle
$$

with $r_{i j}=\left|\mathbf{r}_{i}-\mathbf{r}_{j}\right|$ the distance between particles $i$ and $j$.

Figure 4 shows the radial distribution functions, as obtained from simulation and experiment, for three values of $\phi$ and at each $\phi$ for different values of $\Gamma$. Also included is the $g(r)$ for $\Gamma=0$ for each value of $\phi$, which thus corresponds to the situation where the interactions in the experiment are hard-disk-like and in the simulation the particles interact via the potential given by Eq. (3). In Fig. 4, the distance $r$ on the $x$ axis is scaled by the hard-sphere diameter $\sigma_{\mathrm{HS}}=$ $3.16 \mu \mathrm{m}$ for the experimental data. The simulation data are scaled by the effective diameter $\sigma_{\mathrm{HS}}=1.01 \sigma$ which provides agreement of the fluid equation of state, as obtained with the potential (3), with the one for a hard-disk fluid with hard disks of diameter $\sigma[40,50]$. As can be inferred from Fig. 4, the radial distribution functions as obtained from experiment and simulation are in very good agreement both for $\Gamma=0$ and all the finite values of $\Gamma$ at the different area fractions $\phi$. Note that the small peaks, seen in some of the experimental curves before the main nearest-neighbor peak, are due to the occasional (partly) out-of-plane particles, hence this effect is most visible for the highest $\Gamma$ in Fig. 4(c), i.e., the highest area fraction considered.

The radial distribution functions in Fig. 4 indicate that at each area fraction the nearest-neighbor distance increases with increasing $\Gamma$. Moreover, for all values of $\Gamma$ and $\phi$, the smallest values of $r$ for which $g(r)$ is clearly larger than zero is significantly above $r / \sigma_{\mathrm{hs}}=1.0$, suggesting that hard-core interactions are not relevant for the considered thermodynamic states, which is consistent with our conclusions from considering the equation of state in Sec. IV A. Crucially, this agreement between experiment and simulation indicates that the simulation is fully capturing the direct interactions, which play a crucial role in reducing the long-time self-diffusion coefficient with respect to its value at infinite dilution with increasing $\Gamma$.

\section{Mean-squared displacement and long-time self-diffusion coefficient}

Now, we address the question of whether hydrodynamic interactions (HI) affect the long-time self-diffusion coefficient in our paramagnetic colloidal systems by comparing our experiments to the BD simulations, where $\mathrm{HI}$ are absent. To this end, we consider the mean-squared displacement (MSD) of a tagged particle, $\left\langle\Delta \mathbf{r}^{2}\right\rangle$, which is defined by

$$
\left\langle\Delta \mathbf{r}^{2}(t)\right\rangle=\left\langle\frac{1}{N} \sum_{i}^{N}\left(\mathbf{r}_{i}(t)-\mathbf{r}_{i}(0)\right)^{2}\right\rangle,
$$

where $\langle\cdots\rangle$ corresponds to a time average. In Fig. 5, we show $\left\langle\Delta \mathbf{r}^{2}\right\rangle / \sigma_{\mathrm{hs}}^{2}$ as a function of the scaled time $t D_{0} / \sigma_{\mathrm{hs}}^{2}$. In the three panels of the figure, the MSDs are displayed for the same values of $\phi$ and $\Gamma$ as for the $g(r)$ in Fig. 4, and the insets show the long-time behavior of the MSD more clearly.

First of all, we note that at short times the MSDs for a given $\phi$, but different values of $\Gamma$, all coincide, consistent
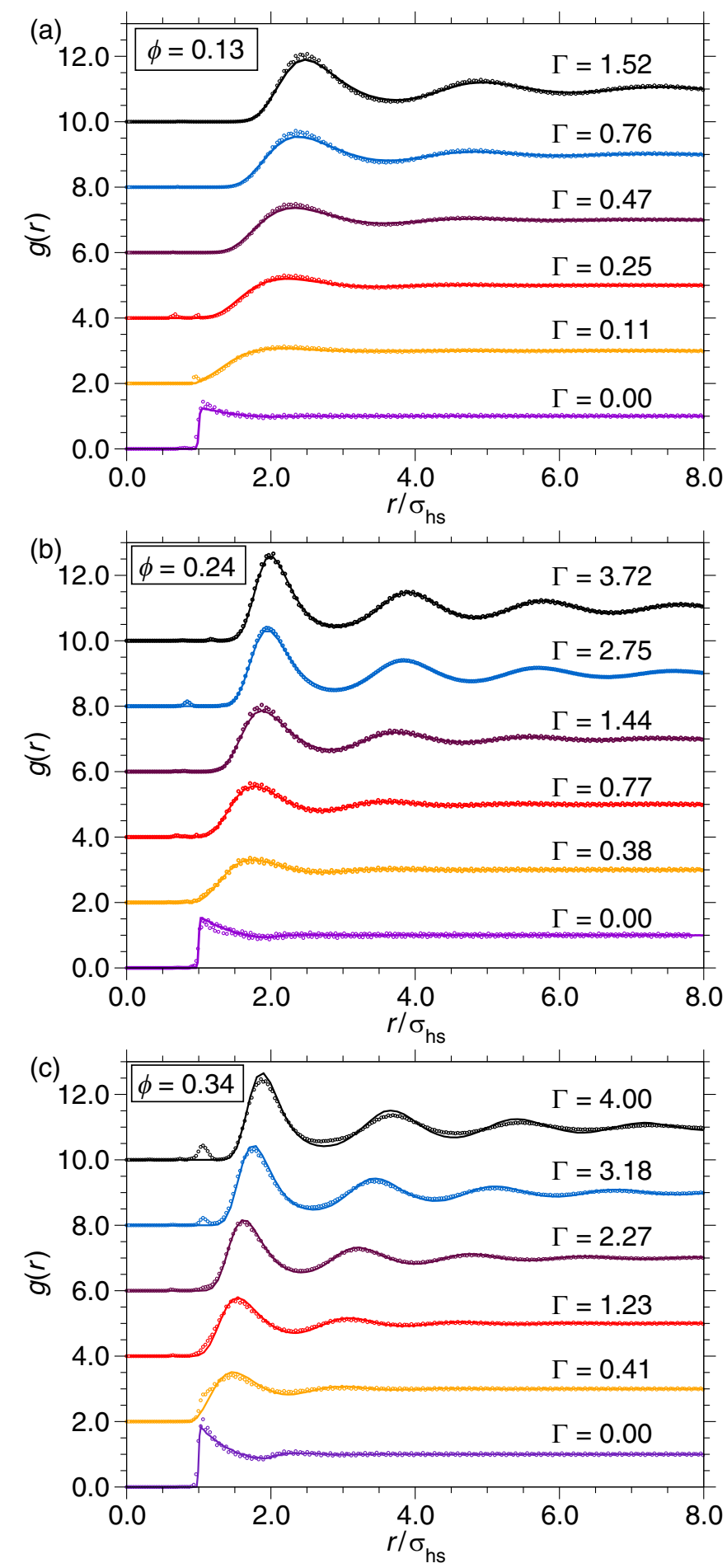

FIG. 4. Radial distribution functions, $g(r)$, for different values of $\Gamma$ and the densities (a) $\phi=0.13$, (b) $\phi=0.24$, and (c) $\phi=0.36$. Experimental and simulation data are shown as symbols and solid lines, respectively. For clarity, the curves in each plot are shifted vertically by constant steps of 2 .

with earlier reports [6]. However, the MSDs at short times are reduced for increasing area fractions in the experiments, an effect that is not observed in the simulations. As in the harddisk situation [14], the deviation between experiment and simulation at short times is attributed to the $\mathrm{HI}$ in the experiment 

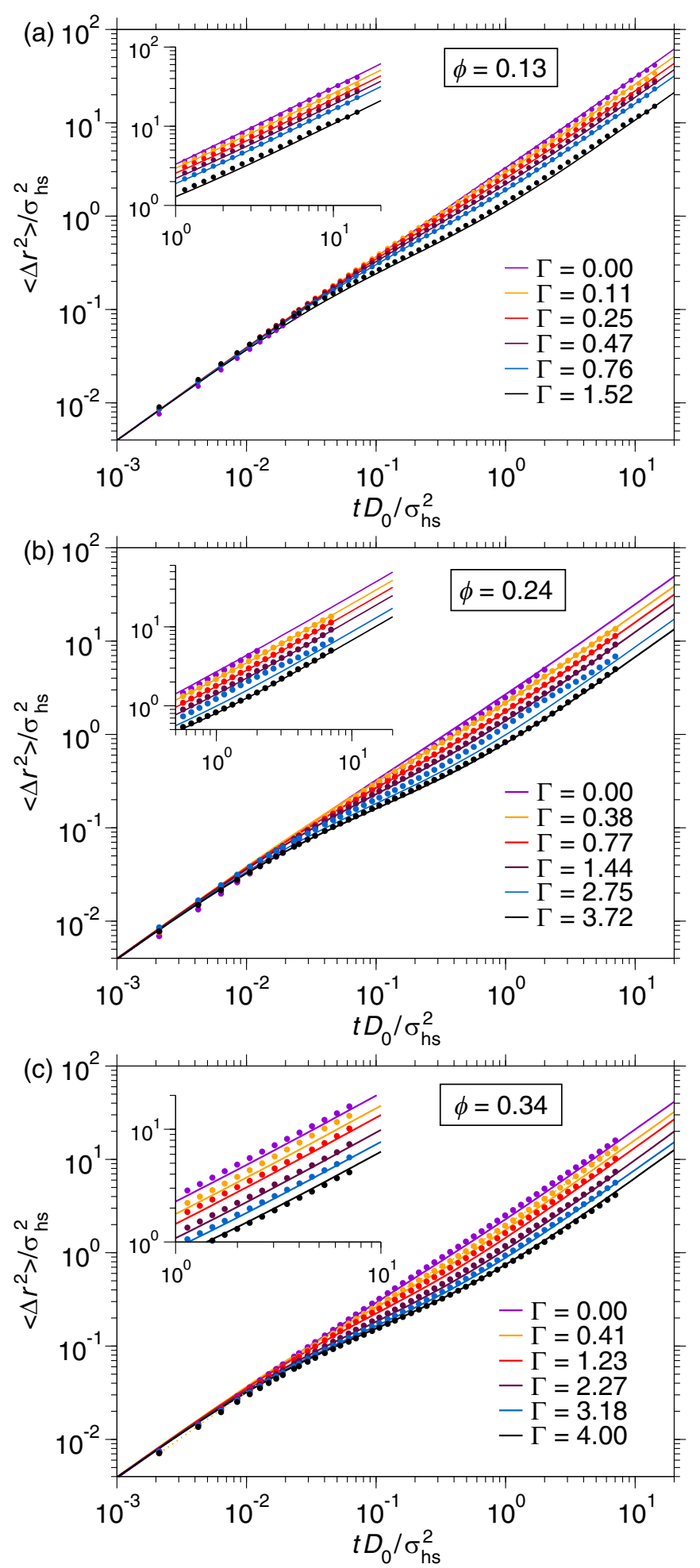

FIG. 5. Scaled mean square displacements, $\left\langle\Delta r^{2}\right\rangle / \sigma_{\mathrm{hs}}$, as a function of scaled time, $t D_{0} / \sigma_{\mathrm{hs}}$, for systems with parameters of Fig. 4.

that are not present in the simulation. Hence, for short times the MSDs of the experiments and simulations agree only in the limit of low area fractions, where $\mathrm{HI}$ are negligible.

This behavior can be quantified by determining the shorttime self-diffusion coefficient, $D_{S}$, from the $t \rightarrow 0$ limit of the MSDs in Fig. 5, and values of $D_{S}$ rescaled by the self-diffusion coefficient at infinite dilution, $D_{0}$, are shown in Fig. 6(a). In our experiments $D_{0}=4.5 \times 10^{-2} \mu \mathrm{m}^{2} / \mathrm{s}$, and this value was obtained by extrapolating $D_{S}$ to $\phi \rightarrow 0$. For all three packing fractions, only relatively small variations in $D_{S}$ are observed as a function of $\Gamma$, particularly when compared to the much larger changes in $D_{L} / D_{0}$, which are also shown. This approximately constant behavior of $D_{S}$ is highlighted by dashed lines indicating the mean value of $D_{S}$ for each packing fraction. Figure 6(a) clearly shows, however, the reduction in the short-time self-diffusion coefficient due to HI with increasing $\phi$. We note that this reduction is small compared to the hard disk study [14], due to the relatively low values of $\phi$ considered here.

The situation is very different in the long-time regime. Here, we find quantitative agreement between the experimental MSD and those obtained from the BD simulations for all area fractions and coupling parameters $\Gamma$ considered. From the MSDs in Fig. 5, we can similarly directly determine the long-time self-diffusion coefficient $D_{\mathrm{L}} / D_{0}$ via the Einstein relation [49] $D_{\mathrm{L}} / D_{0}=\lim _{t \rightarrow \infty}\left\langle\Delta \mathbf{r}^{2}(t)\right\rangle /\left(4 D_{0} t\right)$. These values are also shown in Fig. 6(a), where it is clear that, in contrast to the behavior of the short-time self-diffusion coefficient, $D_{\mathrm{L}} / D_{0}$ decreases substantially with increasing $\Gamma$. The data also clearly demonstrate the good agreement between long-time self-diffusion coefficients from experiment and simulation.

Furthermore, if $\mathrm{HI}$ do not affect the ratio $D_{\mathrm{L}} / D_{0}$, one may expect that the long-time self-diffusion is controlled by the direct interactions and thus there should be an appropriate scaling of $D_{\mathrm{L}} / D_{0}$ with $\Gamma^{2 / 3}$ such that the corresponding reduced long-time self-diffusion coefficient is only a function of the reduced density $\Gamma^{2 / 3}$. To find this reduced self-diffusion coefficient, $D_{\mathrm{L}}^{\star}$, we consider the equations of motion for the BD simulation, Eq. (5). Note that the following conclusions would be exactly the same if we would consider the exact BD equations of motion instead of the discretized ones, as given by Eq. (5). First, we introduce the reduced coordinates $\mathbf{r}_{i}^{\star}$ and time $t^{\star}$ by the equations $\mathbf{r}_{i}=\mathbf{r}_{i}^{\star} \rho^{-1 / 2}$ and $t=t^{\star} \tau$ with

$$
\tau=\frac{1}{D_{0}}\left(\frac{\rho^{-7 / 2}}{\beta A_{\mathrm{dip}}}\right)^{1 / 2} .
$$

The insertion of $\mathbf{r}_{i}^{\star}$ and $t^{\star}$ in Eq. (5) gives the following dimensionless equation of motion for particle $i$ :

$$
\mathbf{r}_{i}^{\star}\left(t^{\star}+\delta t^{\star}\right)=\mathbf{r}_{i}^{\star}\left(t^{\star}\right)+\mathbf{F}_{i}^{\star} \delta t^{\star} \rho^{\star 3 / 4}+\sqrt{2 \delta t^{\star}} \rho^{\star-3 / 8} \boldsymbol{\xi}_{i}
$$

with $\mathbf{F}_{i}^{\star}=\sum_{j \neq i} \frac{1}{r_{i j}^{*}} \frac{\mathbf{r}_{i j}^{*}}{r_{i j}^{*}}$ the reduced force on particle $i$ and $\rho^{\star}=\left(\beta A_{\text {dip }}\right)^{2 / 3} \rho$ the reduced density for $n=3$ and $D=2$, as above. Equation (12) indicates that, as for the pressure, an appropriate scaling of the long-time self-diffusion coefficient $D_{\mathrm{L}}$ leads to a reduced self-diffusion coefficient $D_{\mathrm{L}}^{\star}$ that only depends on the reduced density $\rho^{\star} \equiv \Gamma^{2 / 3}$. The reduced quantity $D_{\mathrm{L}}^{\star}$ can be found via $D_{\mathrm{L}}=D_{\mathrm{L}}^{\star} \frac{1}{\rho \tau}$, using the timescale $\tau$, as defined by Eq. (11). As a result, we obtain

$$
D_{\mathrm{L}}^{\star}\left(\rho^{\star} \equiv \Gamma^{2 / 3}\right)=\frac{D_{\mathrm{L}}}{D_{0} \rho^{\star 3 / 4}}=\frac{D_{\mathrm{L}}}{D_{0} \Gamma^{1 / 2}} .
$$

Note that the use of $\tau$, as introduced in Sec. IV A, leads to a similar result for the reduced long-time diffusion coefficient. 

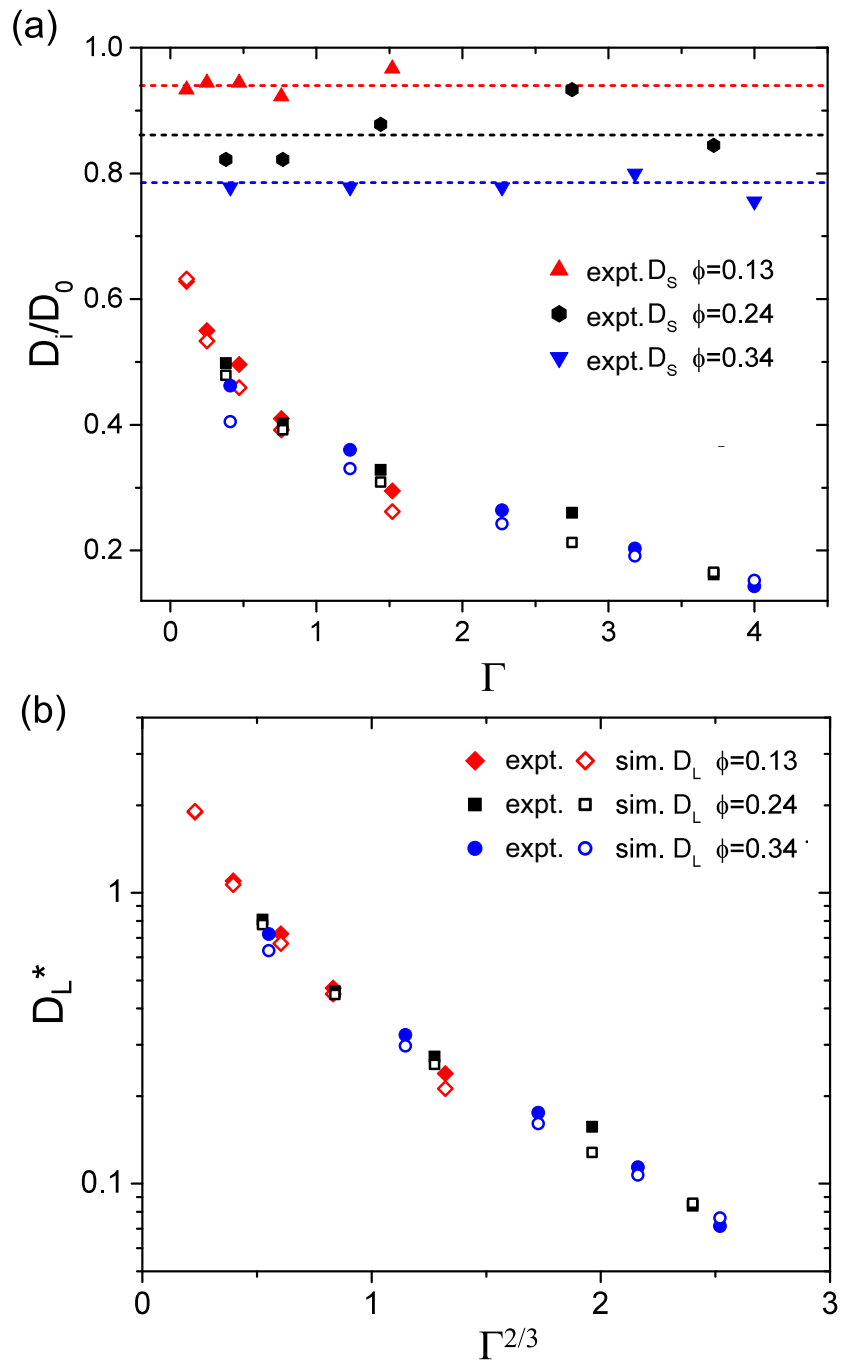

FIG. 6. (a) Long- and short-time self-diffusion coefficients rescaled by the self-diffusion coefficient at infinite dilution, $D_{0}$, as a function of $\Gamma$ for different area fractions. $D_{L}$ is shown for experiments (filled symbols) and simulations (open symbols), as described by the legend in (b), and $D_{S}$ for the experiments only. Dashed lines show the mean value of $D_{S}$ for each area fraction. (b) The reduced long-time self-diffusion coefficient, $D_{\mathrm{L}}^{\star}$, as obtained from the simulations and experiments, is shown as a function of $\Gamma^{2 / 3}$ for different area fractions.

In this case, it is given by $D_{\mathrm{L}}^{\star}=D_{\mathrm{L}} \frac{\beta \rho m}{\Gamma^{1 / 2}}$. The latter reduced diffusion coefficient would be the appropriate quantity in the case of Newtonian dynamics, where the MSD at short times is proportional to $t^{2}$.

Figure 6(b) shows the reduced long-time diffusion coefficient $D_{\mathrm{L}}^{\star}$ from simulation and experiment as a function of $\Gamma^{2 / 3}$ for three different area fractions $\phi$. All the data collapse nicely on one master curve, as predicted by Eq. (13). This clearly indicates that, despite the effect of $\mathrm{HI}$ at short times in the experiment, the reduced long-time self-diffusion coefficient $D_{\mathrm{L}}^{\star}$ is solely a function of $\Gamma^{2 / 3}$ and follows the behavior, as predicted by Eq. (13), for simple BD without taking into account HI. In this sense, the long-time self-diffusion is solely due to the action of direct interactions between the particles.
The picture that thus emerges for our paramagnetic colloidal system considered here is similar to the one recently reported for hard disks [14]. In other words, even in a system with more complex, long-ranged interactions, HI effectively do not affect the long-time self-diffusion coefficient.

We note, however, that this result contrasts with earlier experimental and theoretical studies on similar systems $[6,36,37]$ which proposed that $\mathrm{HI}$ enhance the long-time self-diffusion coefficient $D_{\mathrm{L}}$ in the presence of the longranged $r^{-3}$ interactions between the paramagnetic particles. However, a number of differences between our current study and these earlier studies could account for this disparity. First, compared to the results reported in this work, the experimental data in [6,37] cover a relatively short range of times. As such, their conclusion on the enhancement of $D_{\mathrm{L}} / D_{0}$ due to hydrodynamic interactions applies only to the self-diffusion coefficient in the short and intermediate time regime. Conclusions about the long-time behavior, for example in [37], are drawn from simulation results alone, as the long-time self-diffusion coefficient could not be determined from the experimental data. Our findings for the experimental behavior of the long-time self-diffusion coefficient are therefore drawn from a different time regime than the previous studies. Moreover, the simulation results for the long-time self-diffusion coefficient in [37] do not show the expected decrease in $D_{L}$ with $\phi$, making the observation of an increase in $D_{L}$ with HI in the simulations also difficult to interpret.

With respect to the behavior of the short-time self-diffusion coefficient, our results indicate that hydrodynamic interactions do effect $D_{S}$, in line with previous results [14,16], although, we find no evidence of an enhancement due to HI. We instead observe a decrease in $D_{S}$ with increasing $\phi$ for fixed $\Gamma$ and no change in $D_{S}$ with $\Gamma$ for fixed $\phi$. This different variation in $D_{S}$ could be a consequence of the differences in confining interface between our work and the experiments in [6,37]. We also note, however, that the method used to estimate the self-diffusion coefficient at infinite dilution, $D_{0}$, in Refs. [6,37], is to use the $t \rightarrow 0$ limit of the MSD, i.e., to associate $D_{0}$ with the short-time self-diffusion coefficient $D_{S}$. This approach is chosen as it is reported that over the range of area fractions considered $(\phi \leqslant 0.10)$ no change in this value is observed. However, here we find that even for the lowest packing fraction considered, $\phi=0.13$, there is a small but finite change in $D_{S}$, consistent with previous findings for the reduction of $D_{S}$ for $\phi<0.1$ in hard disks [14]. This suggests that it is inappropriate to rescale by a limiting value of the MSD rather than a more accurate estimation of $D_{0}$.

\section{CONCLUSIONS}

We have investigated the structure and dynamics of twodimensional fluids of particles with long-ranged interactions by comparing Brownian dynamics (BD) simulations to colloidal experiments on paramagnetic particles. In particular, we find that there is quantitative agreement in the reduced longtime self-diffusion coefficient $D_{\mathrm{L}}^{\star}$ between $\mathrm{BD}$ and experiment over a wide range of the reduced density $\Gamma^{2 / 3}$. Crucially, as the BD simulations do not include hydrodynamic interactions, this result indicates that $\mathrm{HI}$ effectively do not affect the 
long-time self-diffusion coefficient in our experimental system of particles with long-ranged interactions.

In making the comparison between experiment and simulation, we emphasize that we have considered states in the experiment where the interactions between the particles are fully controlled by the $r^{-3}$ interactions, i.e., the hard core of the particles is not relevant for any thermodynamic properties. We have inferred this from a careful study of the equation of state in the BD simulations, demonstrating that this shows the scaling behavior expected for an inverse power-law potential for the states considered in the experiments. Furthermore, we have also demonstrated that the radial distribution functions, a key structural quantity, as obtained from the experiment and the BD simulation are in very good agreement. Importantly, this indicates that the direct interactions that act to reduce the value of $D_{\mathrm{L}}^{\star}$ with increasing $\Gamma^{2 / 3}$ are the same in simulation and experiment. As such, the action of these direct interactions alone is sufficient to produce the reduction in the long-time self-diffusion coefficient with increasing $\Gamma$ that we observe in the experiments. We also note that the collapse of the experimental data for $D_{\mathrm{L}}^{\star}$ as a function of $\Gamma^{2 / 3}$ onto a master curve indicates independently from the comparison to the simulations that $\mathrm{HI}$ do not contribute to the long-time selfdiffusion. If HI would affect the long-time self-diffusion, one would expect that the scaling of the long-time self-diffusion coefficient does not work. In this case, $D_{\mathrm{L}}^{\star}$ would not be solely a function of $\Gamma^{2 / 3}$.

Our conclusion that HI effectively do not affect the longtime self-diffusion coefficient of paramagnetic particles is in line with similar experiments on colloidal hard-sphere fluids in quasi-two-dimensional geometry [14]. As such, it would be an interesting topic for forthcoming studies to address the role of $\mathrm{HI}$ on long-time self-diffusion in three-dimensional colloidal suspensions.

\section{ACKNOWLEDGMENTS}

We thank the Royal Society, the EPSRC, the ERC (ERC Starting Grant No. 279541-IMCOLMAT) and the DFG FOR 1394 (Grant No. HO 2231/7-2) for financial support.
[1] K. Zahn, R. Lenke, and G. Maret, Phys. Rev. Lett. 82, 2721 (1999).

[2] K. Zahn and G. Maret, Phys. Rev. Lett. 85, 3656 (2000).

[3] S. Deutschländer, T. Horn, H. Löwen, G. Maret, and P. Keim, Phys. Rev. Lett. 111, 098301 (2013).

[4] M. Engel, J. A. Anderson, S. C. Glotzer, M. Isobe, E. P. Bernard, and W. Krauth, Phys. Rev. E 87, 042134 (2013).

[5] A. L. Thorneywork, J. L. Abbott, D. G. A. L. Aarts, and R. P. A. Dullens, Phys. Rev. Lett. 118, 158001 (2017).

[6] K. Zahn, J. M. Méndez-Alcaraz, and G. Maret, Phys. Rev. Lett. 79, 175 (1997).

[7] K. Zahn and G. Maret, Curr. Opin. Colloid Interface Sci. 4, 60 (1999).

[8] B. X. Cui, B. H. Lin, and S. A. Rice, J. Chem. Phys. 114, 9142 (2001).

[9] B. X. Cui, H. Diamant, B. H. Lin, and S. A. Rice, Phys. Rev. Lett. 92, 258301 (2004).

[10] N. Hoffmann, F. Ebert, C. N. Likos, H. Löwen, and G. Maret, Phys. Rev. Lett. 97, 078301 (2006).

[11] N. Hoffmann, C. N. Likos, and H. Löwen, J. Phys.: Condens. Matter 18, 10193 (2006).

[12] X. G. Ma, W. Chen, Z. R. Wang, Y. Peng, Y. L. Han, and P. E. Tong, Phys. Rev. Lett. 110, 078302 (2013).

[13] A. L. Thorneywork, R. Roth, D. G. A. L. Aarts, and R. P. A. Dullens, J. Chem. Phys. 140, 161106 (2014).

[14] A. L. Thorneywork, R. E. Rozas, R. P. A. Dullens, and J. Horbach, Phys. Rev. Lett. 115, 268301 (2015).

[15] A. L. Thorneywork, D. G. A. L. Aarts, J. Horbach, and R. P. A. Dullens, Soft Matter 12, 4129 (2016).

[16] A. L. Thorneywork, D. G. A. L. Aarts, J. Horbach, and R. P. A. Dullens, Phys. Rev. E 95, 012614 (2017).

[17] A. L. Thorneywork, S. K. Schnyder, D. G. A. L. Aarts, J. Horbach, R. Roth, and R. P. A. Dullens, Mol. Phys. 116, 3245 (2018).

[18] M. D. Carbajal-Tinoco, F. Castro-Román, and J. L. ArauzLara,Phys. Rev. E 53, 3745 (1996).
[19] O. Marnette, E. Perez, F. Pincet, and G. Bryant, Colloids Surf. A 346, 208 (2009).

[20] T. O. E. Skinner, S. K. Schnyder, D. G. A. L. Aarts, J. Horbach, and R. P. A. Dullens, Phys. Rev. Lett. 111, 128301 (2013).

[21] S. K. Schnyder, T. O. E. Skinner, A. L. Thorneywork, D. G. A. L. Aarts, J. Horbach, and R. P. A. Dullens, Phys. Rev. E 95, 032602 (2017).

[22] F. Evers, C. Zunke, R. D. L. Hanes, J. Bewerunge, I. Ladadwa, A. Heuer, and S. U. Egelhaaf, Phys. Rev. E 88, 022125 (2013).

[23] J. Bewerunge, A. Sengupta, R. F. Capellmann, F. Platten, S. Sengupta, and S. U. Egelhaaf, J. Chem. Phys. 145, 044905 (2016).

[24] J. Bewerunge, I. Ladadwa, F. Platten, C. Zunke, A. Heuer, and S. U. Egelhaaf, Phys. Chem. Chem. Phys. 18, 18887 (2016).

[25] H. König, R. Hund, K. Zahn, and G. Maret, Eur. Phys. J. E 18, 287 (2005).

[26] S. Mazoyer, F. Ebert, G. Maret, and P. Keim, Europhys. Lett. 88, 66004 (2009).

[27] C. L. Klix, F. Ebert, F. Weysser, M. Fuchs, G. Maret, and P. Keim, Phys. Rev. Lett. 109, 178301 (2012).

[28] B. Illing, S. Fritschi, D. Hajnal, C. L. Klix, P. Keim, and M. Fuchs, Phys. Rev. Lett. 117, 208002 (2016).

[29] B. Illing, S. Fritschi, H. Kaiser, C. L. Klix, G. Maret, and P. Keim, Proc. Natl. Acad. Sci. USA 114, 1856 (2017).

[30] R. Kurita and E. R. Weeks, Phys. Rev. E 82, 041402 (2010).

[31] S. Vivek, C. P. Kelleher, P. M. Chaikin, and E. R. Weeks, Proc. Natl. Acad. Sci. USA 114, 1850 (2017).

[32] S. Vivek and E. R. Weeks, J. Chem. Phys. 147, 134502 (2017).

[33] A. H. Marcus, J. Schofield, and S. A. Rice, Phys. Rev. E 60, 5725 (1999).

[34] C. A. Murray and D. H. Van Winkle, Phys. Rev. Lett. 58, 1200 (1987).

[35] J. K. G. Dhont, An Introduction to Dynamics of Colloids (Elsevier, Amsterdam, 1996).

[36] G. Nägele and P. Baur, Physica A 245, 297 (1997). 
[37] B. Rinn, K. Zahn, P. Maass, and G. Maret, Europhys. Lett. 46, 537 (1999).

[38] M. Medina-Noyola, Phys. Rev. Lett. 60, 2705 (1988).

[39] J. C. Crocker and D. G. Grier, J. Colloid Interface Sci. 179, 298 (1996).

[40] E. Lange, J. B. Caballero, A. M. Puertas, and M. Fuchs, J. Chem. Phys. 130, 174903 (2009).

[41] A. Grzybowski, E. Gwóźdź, and A. Bródka, Phys. Rev. B 61, 6706 (2000).

[42] D. L. Ermak and Y. Yeh, Chem. Phys. Lett. 24, 243 (1974).

[43] D. L. Ermak, J. Chem. Phys. 62, 4189 (1975).
[44] B. Dünweg and W. Paul, Int. J. Mod. Phys. C 2, 817 (1991).

[45] S. Plimpton, J. Comp. Phys. 117, 1 (1995).

[46] W. G. Hoover, M. Ross, K. W. Johnson, D. Henderson, J. A. Barker, and B. C. Brown, J. Chem. Phys. 52, 4931 (1970).

[47] W. G. Hoover, S. G. Gray, and K. W. Johnson, J. Chem. Phys. 55, 1128 (1971).

[48] Y. Hiwatari, H. Matsuda, T. Ogawa, N. Ogita, and A. Ueda, Prog. Theor. Phys. 52, 1105 (1974).

[49] J.-P. Hansen and I. R. McDonald, Theory of Simple Liquids, 3rd ed. (Academic, London, 2006).

[50] R. Benjamin and J. Horbach, Phys. Rev. E 91, 032410 (2015). 